\title{
Erratum to: MicroRNA 181a improves proliferation and invasion, suppresses apoptosis of osteosarcoma cell
}

\author{
Zhu Jianwei • Liu Fan • Liu Xiancheng • Bai Enzhong • \\ Li Shuai • Li Can
}

Published online: 26 October 2014

(C) International Society of Oncology and BioMarkers (ISOBM) 2014

\section{Erratum to: Tumor Biol. (2013) 34:3331-3337}

DOI 10.1007/s13277-013-0902-0

After the original article published online, it came to authors' attention that a wrong version of Fig. 5 was published with the article due to authors' negligence. The correct version of Fig. 5 and legends appear in the erratum. There is no other change in the results or conclusions of the article.

Additionally, there was an error in the published funding statement. The correct funding is: This study was supported by Natural Science Foundation of Jiangsu Province (Grant No. BK20131199) and The "Six Talent Peaks Program" of Jiangsu Province of China. The authors regret this error.

The online version of the original article can be found at http://dx.doi.org/ 10.1007/s13277-013-0902-0.

Z. Jianwei $(\bowtie) \cdot$ L. Fan $\cdot$ L. Shuai

Department of Orthopedics,

Affiliated Hospital of Nantong University, 20 Xishi Road,

Nantong 226001, Jiangsu Province, People's Republic of China

e-mail: zhujianwei_nt@163.com

L. Xiancheng

Department of Oncology, Affiliated Hospital of Nantong

University, Nantong 226001, Jiangsu Province,

People's Republic of China

B. Enzhong

Department of Orthopedics, Feicheng Hospital of Traditional

Chinese Medicine, Feicheng 271608, Shandong Province,

People's Republic of China

L. Can

Bio-X Center, Shanghai Jiao Tong University, Shanghai 200030,

People's Republic of China 


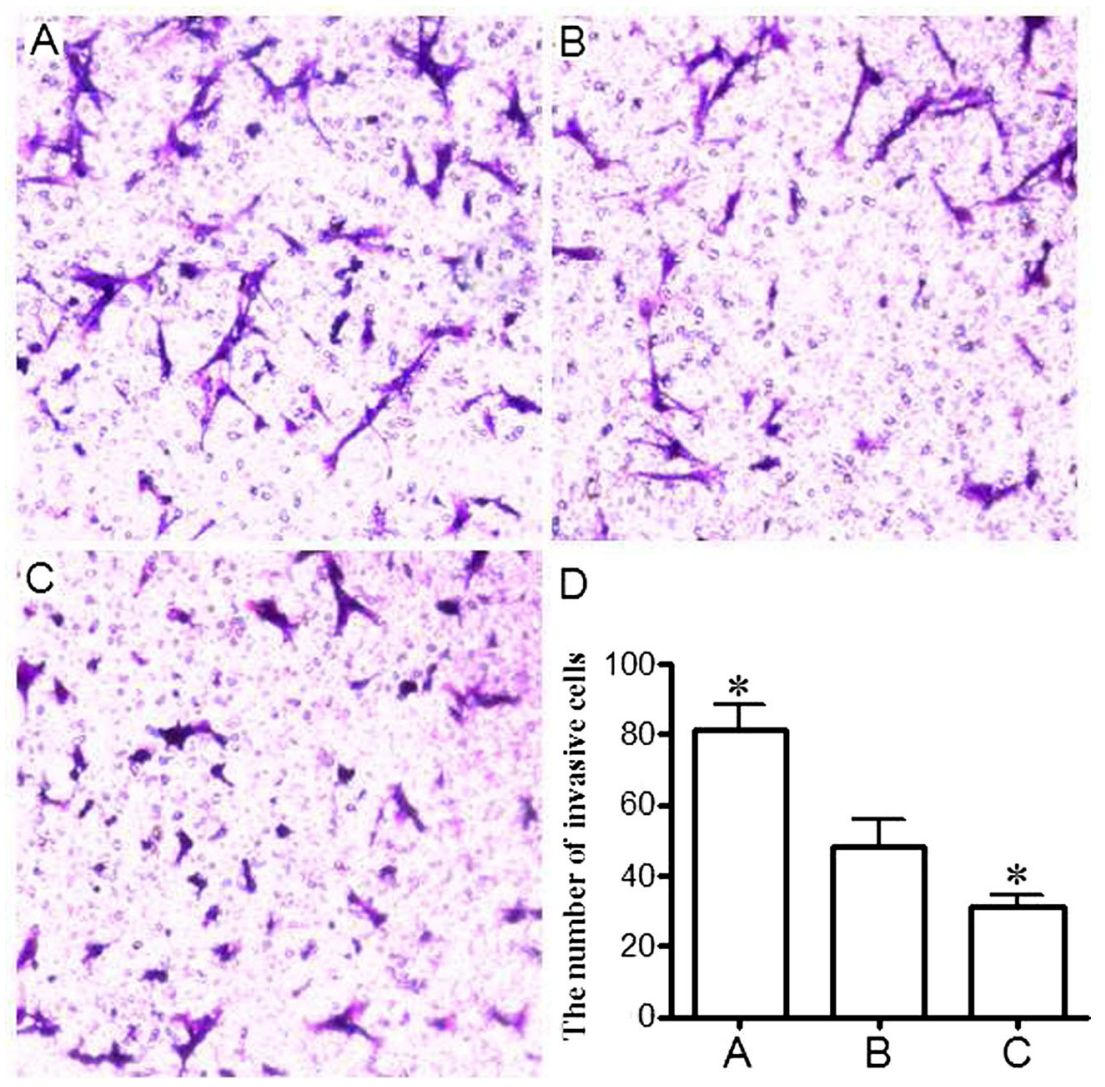

Fig. 5 The effect of miR-181a on MG63 cell invasion ability. a, b, c The crystal violet staining of the MG63 cells that passed through the polycarbonate membrane ( $A$ : overexpression group, $B$ : blank group, $C$ : knockdown group). $\mathbf{d}$ the number of cells passed through transwell invasion chamber $\left({ }^{*} p<0.05\right.$ compared to $\left.B\right)$. These data were analyzed by oneway ANOVA. All experiments were repeated three times with three replicates each 